\title{
Erratum to: Polymer particles having molecule-imprinted skin layer
}

\author{
Hiroshi Ugajin • Norio Ueno • Takashi Oka • \\ Toru Okamoto $\cdot$ Haruma Kawaguchi
}

Published online: 24 April 2013

(C) Springer-Verlag Berlin Heidelberg 2013

Erratum to: Colloid Polym Sci (2013) 291:109-115

DOI 10.1007/s00396-012-2685-z

The original version of the article, unfortunately, contained an error.

The list of authors in the original version was incorrect.

The correct list of authors is given above and their corresponding affiliations are provided below.

The online version of the original article can be found at http://dx.doi.org/ 10.1007/s00396-012-2685-z.

H. Ugajin · H. Kawaguchi

Graduate School of Science and Technology

Keio University, 3-14-1, Hiyoshi,

Yokohama 223-0061, Japan

N. Ueno $\cdot$ T. Oka $\cdot$ T. Okamoto

Research Center, Shiseido Co. Ltd

2-2-1, Hayabuchi, Tsuzuki,

Yokohama 224-8558, Japan

H. Kawaguchi $(\square)$

Graduate School of Engineering, Kanagawa University

3-23-1, Rokkakubashi,

Yokohama 221-8686, Japan

e-mail: haruma@kanagawa-u.ac.jp 